\title{
On the heat in the nozzle of the industrial pneumatic pulsator
}

Received: 2 June 2015 / Revised: 31 October 2015 / Published online: 26 December 2015

(C) The Author(s) 2015. This article is published with open access at Springerlink.com

\begin{abstract}
An industrially applicable nozzle is the subject of this study. The nozzle is an auxiliary equipment of a pneumatic pulsator system for unclogging outlets of silos which store loose materials. The aim is to determine the amount of heat which is generated during one work cycle of the system. Investigation in this field has not been carried out so far, and the present-day designing process is significantly based on heuristic knowledge. The heat is calculated by using results of a numerical simulation. The Finite Volume Method has been used with a thermodynamically ideal gas model. The airflow is assumed to be transient, compressible and supersonic, and it is driven by a time-varying pressure difference. There is an estimation of discretization error of the numerical results carried out in order to confirm the reliability of the solution. The error estimation shows that the results lie in the vicinity of the exact solution of the governing equations. Instantaneous results of the simulation indicate a locally flow which intensifies flow parameters in a similar way as the convergingdiverging nozzles do. The value of the total heat generated during gas conversion within the nozzle is negative; thus, the nozzle could be cooled during its functioning.
\end{abstract}

\section{List of symbols}

$\begin{array}{ll}\mathbf{n} & \text { Normal vector }(-) \\ \mathbf{q} & \text { Heat flux vector }\left(\mathrm{W} / \mathrm{m}^{2}\right) \\ \mathbf{u} & \text { Velocity vector }(\mathrm{m} / \mathrm{s}) \\ C_{\mathrm{p}} & \text { Specific heat at constant pressure }(\mathrm{J} / \mathrm{kg} \mathrm{K}) \\ C_{\mathrm{v}} & \text { Specific heat at constant volume }(\mathrm{J} / \mathrm{kg} \mathrm{K}) \\ E & \text { Total energy }(\mathrm{J}) \\ e & \text { Internal energy of gas }(\mathrm{J} / \mathrm{kg}) \\ h & \text { Mesh size }(-) \\ k & \text { Turbulent energy }(\mathrm{J} / \mathrm{kg}) \\ n & \text { Number of subdivisions }(-) \\ P & \text { Rank of error }(-) \\ p & \text { Pressure }(\text { Pa) } \\ Q & \text { Heat transfer rate }(\mathrm{W}) \\ r & \text { Mesh refinement factor }(-) \\ S & \text { Surface area }\left(\mathrm{m}^{2}\right)\end{array}$

K. J. Wołosz $(\varangle) \cdot$ J. Wernik

Department of Process Equipment, Warsaw University of Technology Plock Branch, Jachowicza 2/4, 09-402 Plock, Poland E-mail: krzysztof.wolosz@ pw.plock.pl Tel.: +48-24-367-5996 


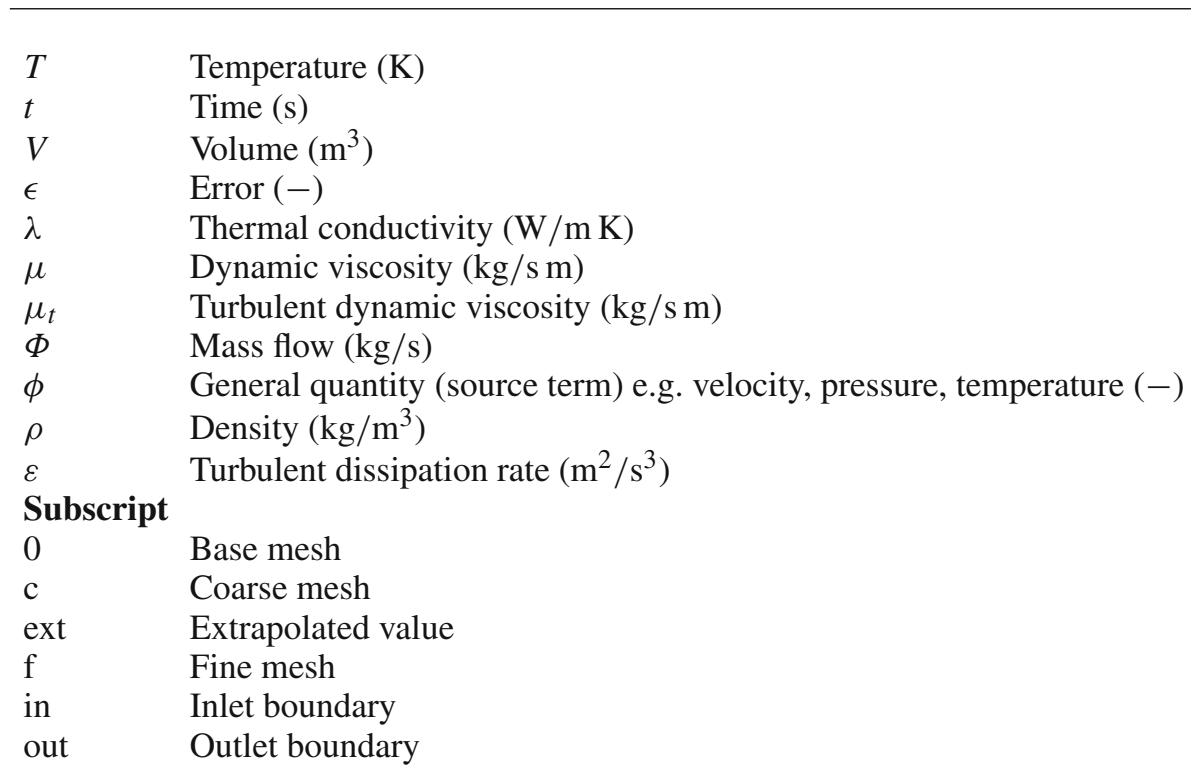

\section{Introduction}

The subject of the study is an industrially applicable nozzle which is an auxiliary equipment of a pneumatic pulsator system. The aim is to determine the amount of the heat which is generated during one work cycle of the system. Results of numerical simulations are used for that purpose.

Pneumatic pulsators are widely used in heavy industry where loose materials are stored and/or transported. The basic function of the pulsators is to destroy an adverse structure in loose and bulk material. Main types of these structures are clinging, bridging, ratholing and arching. A visualization of them is shown in Fig. 1, in which an example location of a pneumatic pulsator system is also presented.

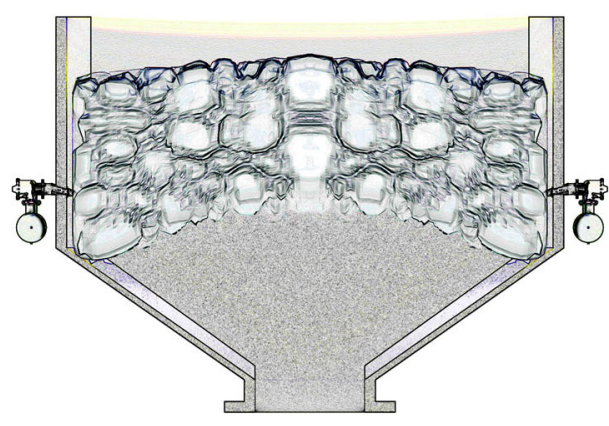

(a)

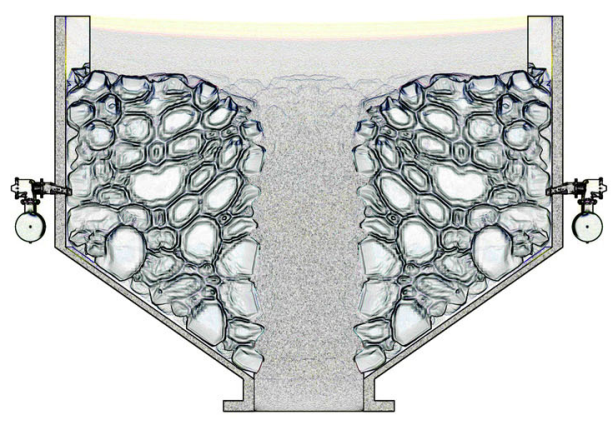

(c)

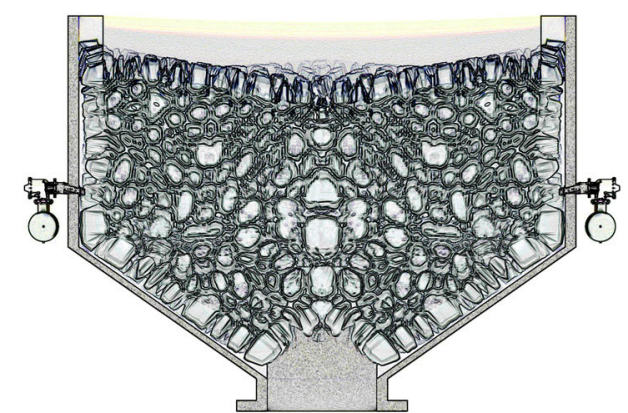

(b)

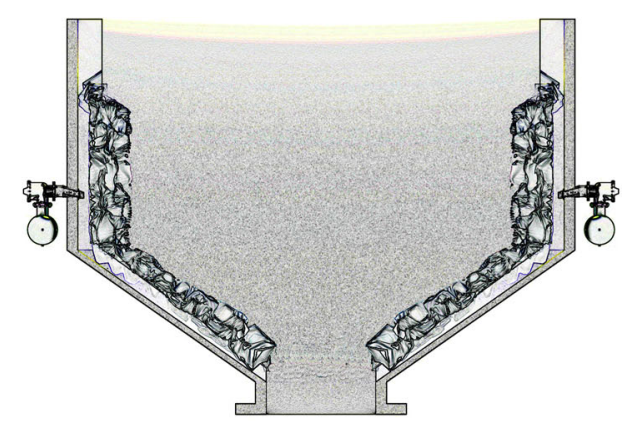

(d)

Fig. 1 Examples of adverse structures of loose material in a silo 
The structures cause stoppages in transportation lines and can provoke a threat to staff. They are specific for loose materials and are formed when cohesion forces are larger than the gravity. In order to destruct them or to prevent them from forming, compressed air is often utilized. The pneumatic pulsators transform energy, which is accumulated in compressed air, to produce a force which acts onto the loose material bed and prevents the cohesion forces from increasing. Due to its source, the force is named a pneumatic impact. In practice, an air blast hits the loose material bed, crushes the adverse structure and moves the material towards the outlet of the silo. Due to the impact, which is induced by air, the pneumatic pulsators are also named air cannons or air blasters.

In some circumstances, the pulsators are equipped in a sort of auxiliary nozzles. The task of the nozzles is to direct the pneumatic impact (directional nozzles) or to protect the pulsator from the heat which can be generated within the silo (heat-resistant nozzle). The nozzles are the last element of the pneumatic pulsator system in the direction of the airflow, and they often have direct contact with the loose material. In numerous applications, both tasks are combined. One of these types of the nozzles is the subject being investigated herein.

The pneumatic pulsator, its head in particular, can be regarded as a quick-release valve. During the pulsator functioning, air is evacuated through the head from the pressure accumulator, thereby emptying it. Air evacuation from the pressure accumulator is a thermodynamic conversion of the gas. The heat generated during this conversion is transported with the airflow and partially transferred onto the walls of the nozzle. A combination of this heat with the one within the silo can cause a failure for the pulsator or the nozzle. The aim of the study is to determine the amount of heat which is generated in the nozzle during one work cycle of the pulsator.

Nozzles have been subjects of studies from many years, and this is a too wide field of activity to be fully presented in this article. However, most researchers investigate nozzles which are applied in the aeronautical or astronautical fields.

The literature review has been started from papers which report analytical and numerical calculations as well as experiments in the field of supersonic flow, excluding flows around airfoils [18]. Supersonic airflow with shock waves can be analytically studied only for rather simple, academic cases. Gas flows using an ideal model of the gas in the case of reflections on simple shapes are investigated in [1], where numerical values are compared to experimental ones. A good practice for shock wave investigation can be found in [5]. This paper describes a flow exiting an open end of a channel, and the numerical results are validated by using an experiment. A supersonic flow in a channel with an open end is also reported in [16]. It is worth to mention that the methods for calculation of compressible flows are still developed [8].

In many cases, a supersonic flow through a converging-diverging nozzle [20], especially through injectors [28], is studied. Investigations of the cavitating flow at high velocities using numerical methods are emphasized in these studies. Many papers consider flows in ejectors. Flow phenomena are reported in scientific studies [4], whereas others consider the influence of the geometry on a specific industrial application [10,29]. Convergingdiverging nozzles are still a subject of investigations due to their wide scope of application [30].

During the literature survey, a deficiency of studies in supersonic airflows which could be applied in heavyindustry equipment has attracted the authors' attention. Furthermore, all aforementioned papers consider flows in closed channels and the considered domains consist of the fluid only inside the channels. Due to the application of the pulsator, the influence on the environment needs to be taken into account.

There was a research and development project undertaken on the pneumatic pulsator in the Department of Process Equipment of Warsaw University of Technology in 2007. The first analysis was published in the report consisting of numerical simulation results in the following year [25]. The phenomenon of vena contracta inside the pneumatic pulsator during the airflow was emphasized in this report. Further work consisting of numerical simulation and optimization of the pulsator was reported in [23]. Computational fluid dynamics (CFD) was also used to predict a fluid-structure interaction (FSI) during the supersonic airflow through the pulsator [26]. The influence of flow parameters on the strength of the pulsator was also considered.

The first attempt to investigate the nozzle of the pulsator was reported in [27], where the combination of the continuous adjoint method and CFD was used to optimize the flow due to pressure drop.

The present article is another step in reporting the development of a whole system for unclogging loose material silos. The subject of the study is a nozzle which is applied in energy and cement plants. The nozzle is basically used to protect the pulsator from the heat in furnaces and silos which store hot contents. Cement industry is a good example where hot pieces of clinker can stick to the wall of the silo.

The nozzle is made of cast iron with the thickness of the walls between 10 and $25 \mathrm{~mm}$. Its visualization is shown in Fig. 2a. The nozzle can be attached to the pneumatic pulsator of nominal outlet diameter $150 \mathrm{~mm}$, and its main dimensions are: length along the flow direction $320 \mathrm{~mm}$, width $487 \mathrm{~mm}$ and height $325 \mathrm{~mm}$. 


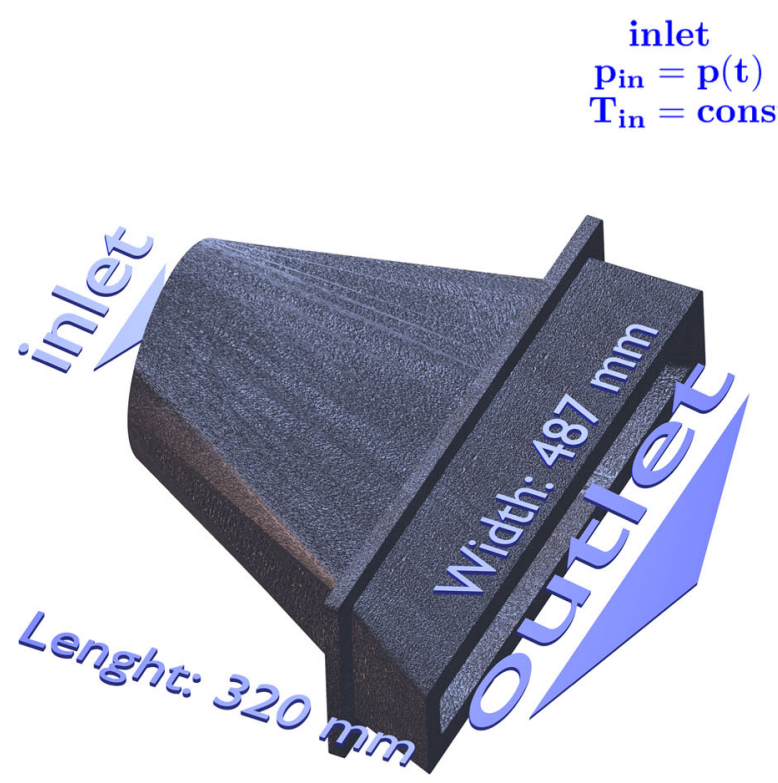

(a)

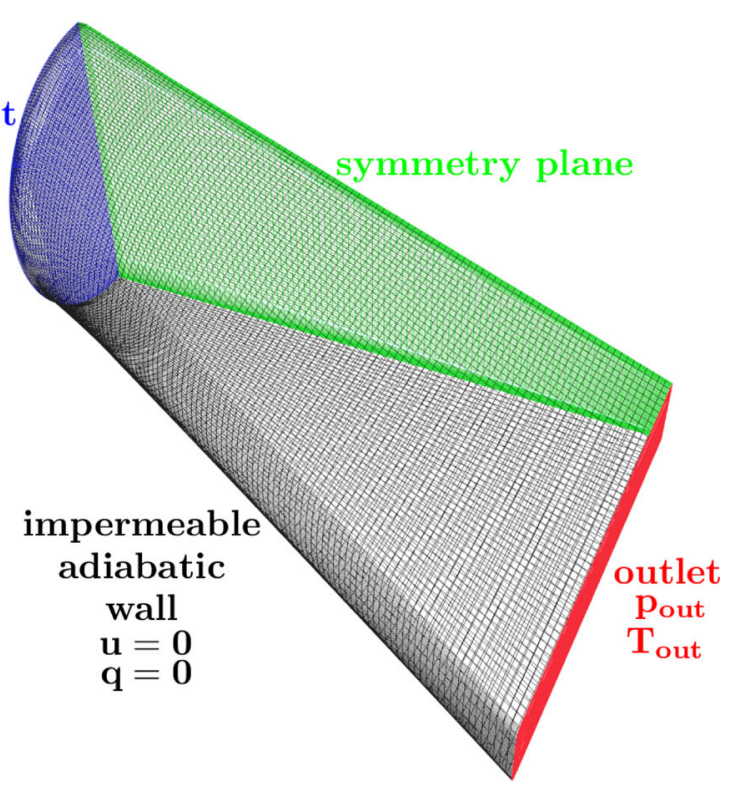

(b)

Fig. 2 The nozzle is being investigated. (a) Visualization with main dimensions, (b) structured mesh with boundary conditions

\section{Numerical model}

The airflow through the pneumatic pulsator is driven by a pressure difference between internal and environmental pressure. The airflow produces the pneumatic impact, which is a force, and by definition, it is a function of momentum and time. Thus, the flow in the pneumatic pulsator is transient. Furthermore, it is regarded as a compressible and a supersonic phenomenon. To calculate the unsteady compressible supersonic flow, the application sonicFoam is used which is part of the OpenFOAM toolbox [15]. The toolbox implements the finite volume method (FVM) which is widely utilized in numerical simulations of fluid flows.

Air is modelled as compressible, thermodynamically ideal gas with invariant gas constant. A very short time of the flow phenomena makes it possible to assume adiabatic flow for solving the governing equations. However, this assumption does not exclude the heat transfer onto the walls of the nozzle if infinitely many work cycles are taken into account.

In their studies of nozzles, many authors use axisymmetric models (e.g., [9]) due to their simplicity and low computational effort. Such a far-reaching simplification of the model will not be applied in this study.

The flow domain is divided into a finite number of cells by using the blockMesh application, which is also a part of the OpenFOAM toolbox. The result of the division is a structured mesh as shown in Fig. $2 \mathrm{~b}$.

\subsection{Governing equations}

The compressible airflow though the nozzle is governed by laws of conservation. Some of them are expressed by the following equations:

- conservation of mass (continuity equation):

$$
\frac{\partial \rho}{\partial t}+\nabla \cdot(\rho \mathbf{u})=0
$$

- conservation of momentum:

$$
\frac{\partial \rho \mathbf{u}}{\partial t}+\nabla \cdot(\rho \mathbf{u u})-\nabla \cdot \mu \nabla \mathbf{u}=-\nabla p
$$

- conservation of energy:

$$
\frac{\partial \rho e}{\partial t}+\nabla \cdot(\rho \mathbf{u} e)-\nabla \cdot\left(\frac{k}{C_{\mathrm{v}}}\right) \nabla e=p \nabla \cdot \mathbf{u}
$$


Equations (1)-(3) must be complemented with equations by which the gas constant (i.e. state equation (4)) and internal energy [Eq. (5)] can be calculated:

$$
\begin{aligned}
R & =C_{\mathrm{p}}-C_{\mathrm{v}}=\frac{p}{\rho T}=287 \mathrm{~J} / \mathrm{kg} \mathrm{K}, \\
e & =C_{\mathrm{v}} T .
\end{aligned}
$$

During the airflow, the heat conduction also proceeds. Thus, an appropriate conduction equation (i.e. Fourier's law) has to be added to the system of equations:

$$
\mathbf{q}=-\lambda \nabla T .
$$

In order to solve the aforementioned equations by using FVM, Gauss linear integration is used, which is second-order accurate, for spacial discretization. To discretize the time derivatives, an implicit Euler, firstorder accurate, scheme is used.

\subsection{Turbulence model}

The system of governing equations delivers results just for laminar flows. For turbulent flows while no turbulence is modelled, the direct numerical simulation (DNS) can be applied [13,14]. DNS is recently often utilized in scientific research, but it requires a very dense mesh; thus, it is hardly ever used in industrial applications because of the high computational effort. For the case described in this article, where $R e=1.5 \times 10^{6}$, a mesh size of over 80 billion of cells would have had to be applied if DNS had been used [19]. Therefore, a turbulence model is needed to deliver accurate results.

A suitable turbulence model can be chosen from many available, but to produce reliable results several factors have to be taken into account. The two-equation model standard k- $\varepsilon$ [12] has been chosen for the investigated case. The name of the model is taken from the equations which describe kinetic energy of turbulence $(\mathrm{k})$ and dissipation rate of this energy $(\varepsilon)$. From the many advantages of using this model, two have to be mentioned. First, this model is suitable when flow does not separate, and second, it creates good results when the parameters of free stream are more important than the influence of the fluid onto the walls [17,22]. The equations of the $\mathrm{k}-\varepsilon$ model are added to the governing equation with some buoyant terms neglected:

$$
\begin{aligned}
\frac{\partial}{\partial t}(\rho k)+\nabla \cdot(\rho k \mathbf{u}) & =\nabla \cdot\left[\left(\mu+\frac{\mu_{t}}{\sigma_{k}}\right) \nabla k\right]+P_{k}-\rho \varepsilon-Y_{M}+S_{k}, \\
\frac{\partial}{\partial t}(\rho \varepsilon)+\nabla \cdot(\rho \varepsilon \mathbf{u}) & =\nabla \cdot\left[\left(\mu+\frac{\mu_{t}}{\sigma_{\varepsilon}}\right) \nabla \varepsilon\right]+C_{1 \varepsilon} \frac{\varepsilon}{k} P_{k}-C_{2 \varepsilon} \rho \frac{\varepsilon^{2}}{k}+S_{\varepsilon}, \\
\mu_{t} & =\rho C_{\mu} \frac{k^{2}}{\varepsilon},
\end{aligned}
$$

where $C_{1 \varepsilon}=1.44, C_{2 \varepsilon}=1.92, C_{\mu}=0.09, \sigma_{\varepsilon}=1.3, \sigma_{k}=1.0$ are model constants. The values of these constants are taken from experiments, and they are well validated in practical applications [22]. $P_{k}$ represents the generation of turbulence kinetic energy due to the mean velocity gradients, $P_{k}=-\rho \overline{\mathbf{u}^{\prime} \mathbf{u}^{\prime}} \nabla \mathbf{u}$, where $\mathbf{u}^{\prime}$ is a vector of velocity fluctuation and the term $\overline{\mathbf{u}^{\prime} \mathbf{u}^{\prime}}$ delivers the mean value of the tensor product. Equations (7)

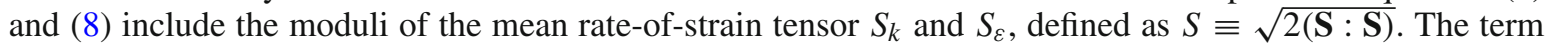
$\mathbf{S}: \mathbf{S}$ gives the scalar product of the rate-of-strain tensor. In Eq. (7), the term $Y_{M}$ denotes viscous destruction and is calculated by $Y_{M}=2 v \overline{\left(\nabla^{2} \mathbf{u}^{\prime}\right)^{2}}$.

\subsection{Boundary conditions}

In order to solve the system of governing equations, a numerical model needs to be complemented by boundary and initial conditions. Time-varying conditions are modelled by using variable inlet pressure. It increases linearly from $0.1 \mathrm{MPa}$ at the start of the simulation up to $0.6 \mathrm{MPa}$ at time $0.02 \mathrm{~s}$. The outlet pressure (and other terms) is calculated according to wave transmissive boundary condition, which is of the Robin type [24], as follows:

$$
\frac{\partial \phi}{\partial t}+\mathbf{u}(\mathbf{n} \cdot \nabla \phi)=\frac{\mathbf{u}}{l_{\infty}}\left(\phi_{\infty}-\phi\right),
$$


where the values of outlet terms $\phi$ are calculated by using far field values $\phi_{\infty}$ being located in a known distance from the outlet $l_{\infty}$. The wave transmissive boundary condition has been applied at the outlet for scalar fields of pressure $\left(p_{\infty}=10^{5} \mathrm{~Pa}\right)$ and temperature $\left(T_{\infty}=300 \mathrm{~K}\right)$ and for velocity vector field $\left(\mathbf{u}_{\infty}=0 \mathrm{~m} / \mathrm{s}\right)$. All the outlet terms with the wave transmissive boundary condition applied have $l_{\infty}=10 \mathrm{~m}$ assumed. The wave transmissive boundary condition minimizes the number of cells in the numerical mesh. The values obtained by using this condition are "transferred" from the outlet of the nozzle to the environment. Furthermore, it does not allow shock waves to be reflected back to the flow domain, what is possible with Neumann-type boundary conditions.

The inlet boundary conditions are Dirichlet type for scalars (pressure and temperature) and Neumann type for velocity; i.e. $\mathbf{n} \cdot \nabla \mathbf{u}=0$.

There is a symmetrical flow assumed in the numerical model; thus, there is only one half of the nozzle analysed with the symmetry plane boundary condition applied. This condition equalizes the components of the gradients which are normal to the plane to zero; i.e. $\mathbf{n} \cdot \nabla \phi=0$.

\section{Heat generated}

When the air flows through the nozzle, there is a gas conversion in which heat is generated. This heat can be transferred onto the walls of the nozzle during infinitely many consecutive work cycles. The heat is determined according to the energy balance [21], and its increment is the result of delivered heat and external work as follows:

$$
\frac{\mathrm{d} E}{\mathrm{~d} t}=H+L,
$$

where $H$ is the sum of the heat exchanged with the environment and the heat of physiochemical reactions in a time unit, and $L$ is the work exchanged with the environment in a time unit.

The derivative of energy is:

$$
\frac{\mathrm{d} E}{\mathrm{~d} t}=\frac{\partial}{\partial t} \int_{V} \rho\left(e+\frac{1}{2} \mathbf{u} \cdot \mathbf{u}\right) \mathrm{d} V+\oint_{S} \rho\left(e+\frac{1}{2} \mathbf{u} \cdot \mathbf{u}\right) \mathbf{u} \cdot \mathbf{n} \mathrm{d} S .
$$

The gas conversion is assumed adiabatic in the absence of internal heat sources (no chemical reactions). Thus:

$$
\frac{\mathrm{d} E}{\mathrm{~d} t}=\oint_{S} \rho\left(e+\frac{1}{2} \mathbf{u} \cdot \mathbf{u}\right) \mathbf{u} \cdot \mathbf{n} \mathrm{d} S
$$

Calculation of the generated heat is based on mean values of source quantities (source terms) which are taken from the values at the inlet and the outlet of the nozzle. The mean values for scalar fields and vector volume fields are obtained according to the following equation:

$$
\bar{\phi}_{S}=\frac{1}{S} \int_{S} \phi \mathrm{d} S .
$$

Mass flux of the gas is a scalar surface field and is determined for each boundary cell. Because the walls are impermeable, only the fluxes of the inlet and the outlet are taken into account and they are obtained by summing cell fluxes of the inlet and the outlet. Because the symmetry plane boundary condition is applied, the mass flux must be doubled:

$$
\Phi=2 \int_{S} \rho \mathbf{u} \cdot \mathbf{n} \mathrm{d} S
$$

The mean values of scalar fields and vector volume fields are not influenced by the symmetry plane boundary condition.

The instantaneous heat generated during the gas conversion in the nozzle is calculated by using Eqs. (13) and (15) with impermeability of the walls taken into account:

$$
Q=\frac{\mathrm{d} E}{\mathrm{~d} t}=\left(\frac{\mathrm{d} E}{\mathrm{~d} t}\right)_{\text {in }}-\left(\frac{\mathrm{d} E}{\mathrm{~d} t}\right)_{\text {out }}=\Phi_{\text {in }}\left(e+\frac{1}{2} \mathbf{u} \cdot \mathbf{u}\right)_{\text {in }}-\Phi_{\text {out }}\left(e+\frac{1}{2} \mathbf{u} \cdot \mathbf{u}\right)_{\text {out }} .
$$

The heat primarily depends on the velocity vector field. This is an important conclusion in order to perform the error estimation properly. 
Table 1 Convergence history for normalized residuals

\begin{tabular}{lll}
\hline Source quantity $\phi$ & Conv. crit. & Final residual (min-max) \\
\hline$p$ & $1 \times 10^{-8}$ & $6.20 \times 10^{-10}-3.84 \times 10^{-9}$ \\
$\mathbf{u}$ & $1 \times 10^{-5}$ & $1.8 \times 10^{-7}-6.8 \times 10^{-6}$ \\
$e$ & $1 \times 10^{-5}$ & $6.544 \times 10^{-8}-9.997 \times 10^{-6}$ \\
$k$ & $1 \times 10^{-8}$ & $1.15 \times 10^{-10}-9.90 \times 10^{-9}$ \\
$\varepsilon$ & $1 \times 10^{-8}$ & $5.90 \times 10^{-11}-9.97 \times 10^{-9}$ \\
\hline
\end{tabular}

\section{Error estimation}

FVM is an iterative method which uses discretization of the continuous fluid domain. Thus, the continuous quantities are approximated by discrete ones and an error of approximation is a permanent element of the method. This error should be estimated to indicate "how far" the numerical results lay from the exact solution.

The estimation of the discretization error must be followed by a verification if none of the gross errors are made. The first step is a checking of the convergence history for all time steps. Table 1 presents the reached convergence (final residual) with convergence criteria. If any of the values of the final residuals is larger than the corresponding convergence criterion, the gross error is made and the numerical model has to be corrected. Formulae for the residual calculations are reported in [6,7], where a deeper knowledge on numerical methods, discretization schemes and error estimation can be found. To estimate the discretization error, a procedure described in [3], and references therein, has been utilized.

The generated heat obtained according to Eq. (16) is mostly influenced by velocity. Hence, this quantity has been chosen for estimating the discretization error. The procedure of estimation requires results of additional simulations by using finer and coarser mesh than the basic one. The refinement factor is a ratio of mean cell sizes between finer-to-basic and basic-to-coarser meshes. For the investigated case, the factors are, respectively, $r_{\mathrm{f}}=n_{\mathrm{f}} / n_{0} \approx 1.3$ and $r_{\mathrm{c}}=n_{0} / n_{\mathrm{c}} \approx 1.33$.

The error order is calculated iteratively by using the following equation:

$$
\begin{aligned}
P & =\frac{1}{\log (r)}\left|\frac{\epsilon_{\mathrm{c}}}{\epsilon_{\mathrm{f}}}+q(P)\right|, \\
q(P) & =\log \left(\frac{r_{\mathrm{f}}^{P}-s}{r_{\mathrm{c}}^{P}-s}\right), \\
s & =\operatorname{sign}\left(\frac{\epsilon_{\mathrm{c}}}{\epsilon_{\mathrm{f}}}\right),
\end{aligned}
$$

where absolute and relative errors are obtained, respectively:

$$
\begin{aligned}
\epsilon_{\mathrm{f}} & =\phi_{\mathrm{f}}-\phi_{0}, \\
\epsilon_{\mathrm{c}} & =\phi_{0}-\phi_{\mathrm{c}}, \\
\epsilon_{\mathrm{rf}} & =\left|\frac{\phi_{\mathrm{f}}-\phi_{0}}{\phi_{\mathrm{f}}}\right|, \\
\epsilon_{\mathrm{rc}} & =\left|\frac{\phi_{0}-\phi_{\mathrm{c}}}{\phi_{0}}\right| .
\end{aligned}
$$

Aforementioned results allow to deliver the value of grid convergence index (GCI) by using the following equations:

$$
\begin{aligned}
\mathrm{GCI}_{\mathrm{f}} & =\frac{1.25 \epsilon_{\mathrm{rf}}}{r_{\mathrm{f}}^{P}-1}=0.19 \times 10^{-3}, \\
\mathrm{GCI}_{\mathrm{c}} & =\frac{1.25 \epsilon_{\mathrm{rc}}}{r_{\mathrm{c}}^{P}-1}=1.05 \times 10^{-3} .
\end{aligned}
$$

The values of GCI make it possible to qualify, beyond the discretization error, the correctness of the model. If the value of GCI for a coarse mesh is large than the one for a fine mesh $\mathrm{GCI}_{\mathrm{c}}>\mathrm{GCI}_{\mathrm{f}}$, it means that the results for the fine mesh are more reliable [2], which confirms the model correctness. 

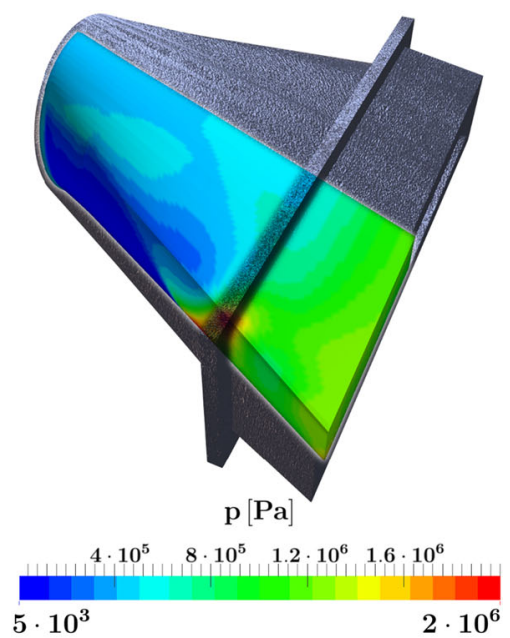

(a)

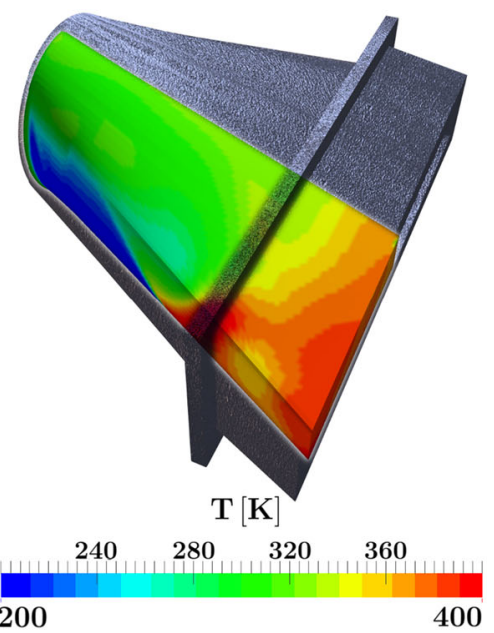

(b)

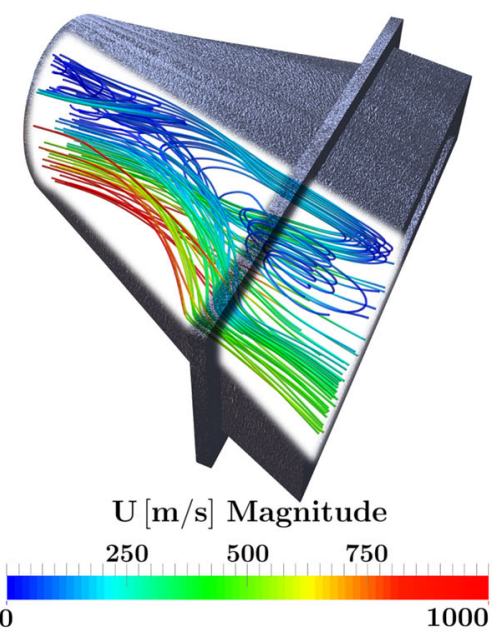

(c)

Fig. 3 Visualization of the results at the time of process $t=0.015 \mathrm{~s}$. a Pressure, $\mathbf{b}$ temperature, $\mathbf{c}$ pathlines 


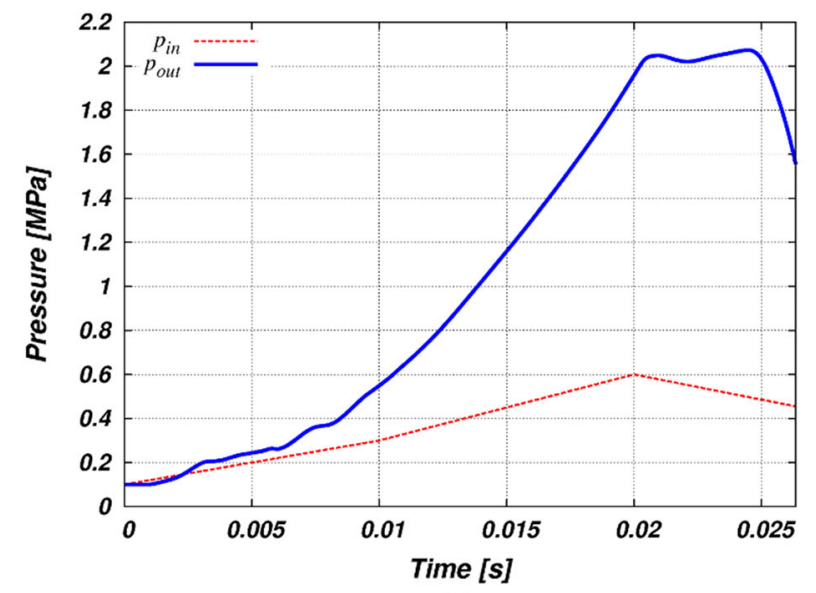

(a)

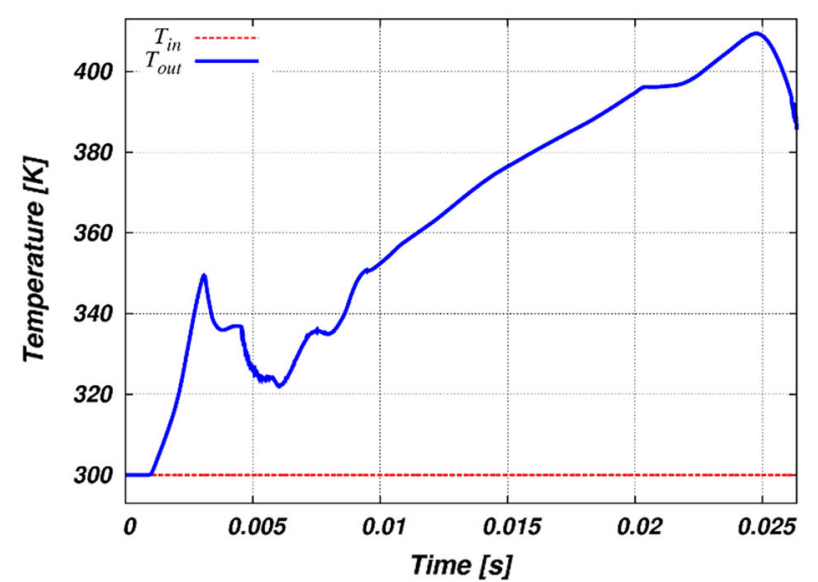

(b)

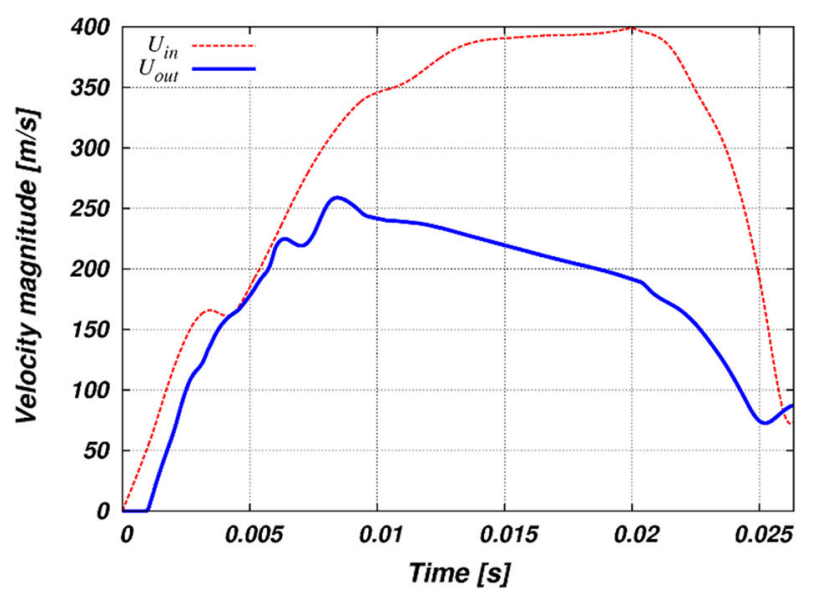

(c)

Fig. 4 Inlet and outlet mean results of source terms. a Pressure, $\mathbf{b}$ temperature, $\mathbf{c}$ velocity

\section{Results and discussion}

The results of the simulation are scalar and vector fields. The visualization of the instantaneous results for simulation time $t=0.015 \mathrm{~s}$ is shown in Fig. 3. Scalar fields, i.e. pressure and temperature, are shown in Fig. 3a 


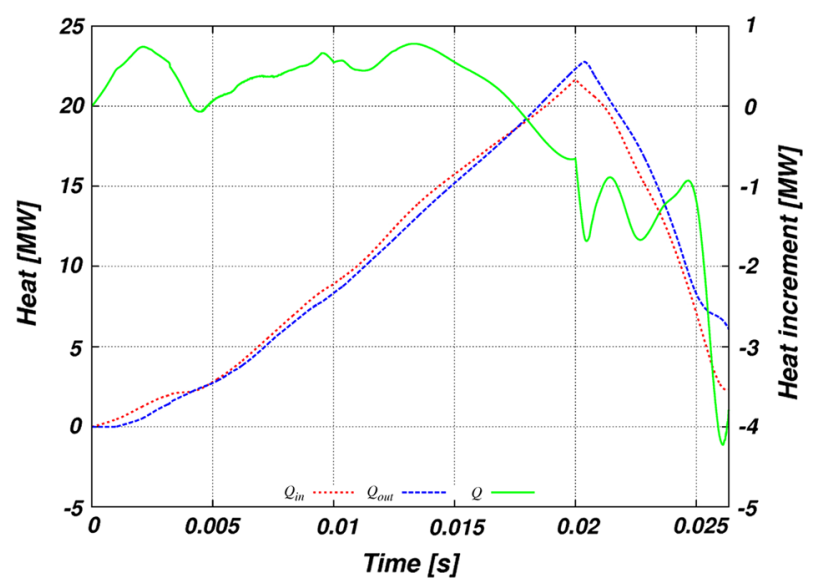

Fig. 5 Heat in the nozzle is changing in time

and $b$, respectively. Figure $3 c$ presents the volume vector field of velocity, which is visualized by stream lines which are coloured by velocity magnitude. The results presented in the visualization can only be considered for quality analysis; however, an interesting phenomenon can be noticed in the results of the velocity field. A vortex created near the symmetry plane denotes a regressive flow occurrence. The possible explanation is that there is a locally chocked flow. For flow of an ideal gas, the Mach number Ma $=\mathbf{u} / \sqrt{\gamma R T}$ depends on velocity and temperature, and chocked flow occurs for a Mach number near 1.

Transient analysis is carried out by using the graphs shown in Fig. 4. Each of the graphs uses results of the simulation at the inlet and the outlet of the nozzle which are averaged by using Eq. (14). It can be noticed that there is an increase in the pressure (Fig. 4a) and the temperature (Fig. 4b) at the outlet, while the velocity (Fig. 4c) decreases at the same time. The maximal outlet pressure is over twice as large as the maximal inlet pressure. During the high compression of the gas, the temperature at the outlet increases to over $400 \mathrm{~K}$ and is much larger than the temperature of delivered air. That proofs the locally chocked flow.

The results of the simulation are utilized to calculate the heat rates delivered to $\left(Q_{\text {in }}\right)$ and received from ( $\left.Q_{\text {out }}\right)$ the nozzle by using Eq. (13). The difference between the heat rates gives a heat increment which is the value of the rate of the heat generated during the gas conversion within the nozzle. The heat increment for each time step is calculated by using Eq. (16) and is shown in Fig. 5. Values of the heat rates are strictly related to velocity and temperature changes. It can be noticed that the values of delivered and released heat rates increase when the pressure raises and they decrease steeply when the pressure drops. The heat increment has no such clear trend. However, while the heat rates $Q_{\text {in }}$ and $Q_{\text {out }}$ decrease, the heat increment reaches values below zero. Therefore, the gas conversion could be divided into two periods. The division could be defined by the moment in time when the heat increment drops below zero. This moment is at the process time of about $17 \mathrm{~ms}$. During the first period, the nozzle is slightly heated because the heat increment has positive values. In contrast, the nozzle is cooled during the last period.

In order to get the information whether the nozzle is heated or cooled during the whole process, the total heat must be determined. In terms of definition of the heat rate, the total heat is an integral of the heat rate over the time. The integral is switched to a sum if the discrete data are taken into account. For the constant time step $\Delta t=10^{-5}$, the total heat is calculated as follows:

$$
\begin{aligned}
& E=\int_{t_{0}}^{t_{1}} Q \mathrm{~d} t=\sum Q \cdot \Delta t \\
& E=-3704.2 J
\end{aligned}
$$

The negative value means that the total delivered heat is smaller than the total heat which is transferred through the outlet of the nozzle. Thus, the results of the simulation indicate that the gas conversion within the nozzle is exothermic. 


\section{Concluding remarks}

Numerical simulation of the supersonic compressible airflow has been carried out, and the results have been estimated and presented in the article. A thermodynamically ideal model of the gas was applied. Provided that the value of the temperature does not exceed $420 \mathrm{~K}$, the model of the gas is not erroneous [11].

Instantaneous results of the simulation have indicated a locally flow which intensifies the flow parameters in a similar way the converging-diverging nozzles do.

The investigation has been aimed at developing and applying a method to calculate the heat which is generated during the gas conversion. The investigated subject is the nozzle which is an auxiliary equipment of the pneumatic pulsator for unclogging the outlets of the silos for loose materials. The method has been intended to use in further investigation on the other elements of the pneumatic pulsator system. The generated heat has been calculated on the basis of the numerical simulation results. The negative value of the heat indicates that the nozzle would be cooled during infinitely many work cycles. Considering the location and application of the nozzle in furnaces and silos with high temperature processes, this is a positive aspect because of additional protection of the pulsator against thermal deformations.

Open Access This article is distributed under the terms of the Creative Commons Attribution 4.0 International License (http:// creativecommons.org/licenses/by/4.0/), which permits unrestricted use, distribution, and reproduction in any medium, provided you give appropriate credit to the original author(s) and the source, provide a link to the Creative Commons license, and indicate if changes were made.

\section{References}

1. Ben-Dor, G.: Shock Wave Reflection Phenomena. Springer, Berlin Heidelberg (2007)

2. Ferziger, J.H., Peric, M.: Computational Methods for Fluid Dynamics. Springer, Berlin (2002)

3. Freitas, C.J.: The issue of numerical uncertainty. In: Second International Conference on CFD in the Minerals and Process Industries CSIRO, pp. 29-34. Melbourne, Australia (1999)

4. Hemidi, A., Henry, F., Leclaire, S., Seynhaeve, J.M., Bartosiewicz, Y.: CFD analysis of a supersonic air ejector. Part I: experimental validation of single-phase and two-phase operation. Appl. Therm. Eng. 29(8), 1523-1531 (2009). doi:10. 1016/j.applthermaleng.2008.07.003

5. Honma, H., Ishihara, M., Yoshimura, T., Maeno, K., Morioka, T.: Interferometric CT measurement of three-dimensional flow phenomena on shock waves and vortices. Shock Waves 13, 179-190 (2003)

6. Jasak, H.: Error analysis and estimation for the finite volume method with applications to fluid flows. Ph.D. thesis, Imperial College of Science, Technology and Medicine, London (1996)

7. Jasak, H., Gosman, A.D.: Element residual error estimate for the finite volume method. Comput. Fluids 32(2), 223-248 (2003). doi:10.1016/S0045-7930(02)00004-X

8. Jing, H., Ru, L., Yaling, H., Zhiguo, Q.: Solutions for variable density low mach number flows with a compressible pressurebased algorithm. Appl. Therm. Eng. 27(11), 2104-2112 (2007). doi:10.1016/j.applthermaleng.2006.11.010

9. Lee, K.H., Setoguchi, T., Matsuo, S., Kim, H.D.: The effect of the secondary annular stream on supersonic jet. KSME Int. J. 17(11), 1793-1800 (2003). doi:10.1007/BF02983610

10. Lin, C., Cai, W., Li, Y., Yan, J., Hu, Y., Giridharan, K.: Numerical investigation of geometry parameters for pressure recovery of an adjustable ejector in multi-evaporator refrigeration system. Appl. Therm. Eng. 61(2), 649-656 (2013). doi:10.1016/j. applthermaleng.2013.08.033

11. Lunev, V.: Real Gas Flows with High Velocities. CRC Press, Boca Raton (2009)

12. Menter, F.R.: Two-equation eddy-viscosity turbulence models for engineering applications. AIAA J. 32(8), 1598-1605 (1994)

13. Moin, P., Mahesh, K.: Direct numerical simulation: a tool in turbulence research. Ann. Rev. Fluid Mech. 30(1), 539-578 (1998). doi:10.1146/annurev.fluid.30.1.539

14. Moser, R., Kim, J., Mansour, N.: Direct numerical simulation of turbulent channel flow up to $\operatorname{Re}=590$. Phys. Fluids 11(4), 943-945 (1999)

15. OpenFOAM: the open source computational fluid dynamics (CFD) toolbox (2013). http://www.openfoam.com/

16. Ota, M., Udagawa, S., Inage, T., Maeno, K.: Interferometric measurement in shock tube experiments. In: InterferometryResearch and Applications in Science and Technology, Ivan Padron (Ed.). InTech (2012)

17. Petrila, T., Trif, D.: Basics of Fluid Mechanics and Introduction to Computational Fluid Dynamics. Springer, Boston (2005)

18. Pirumov, U.: Analytical study of supersonic isentropic flow in a channel of constant cross section and an adjacent laval nozzle. comparison with numerical and experimental results. Fluid Dyn. 29(4), 0-9 (1994)

19. Pozrikidis, C.: Fluid Dynamics: Theory, Computation, and Numerical Simulation. Springer, Boston (2009)

20. Quaatz, J.F., Giglmaier, M., Hickel, S., Adams, N.A.: Large-eddy simulation of a pseudo-shock system in a laval nozzle. Int. J. Heat Fluid Flow 49, 108-115 (2014). doi:10.1016/j.ijheatfluidflow.2014.05.006

21. Szumowski, A., Selerowicz, W., Piechna, J.: Gas Dynamics (in Polish). WUT Publishing, Warsaw (1988)

22. Tu, J., Yeoh, G.H., Liu, C.: Computational Fluid Dynamics. A Practical Approach. Elsevier, Amsterdam (2008) 
23. Urbaniec, K., Wernik, J., Wołosz, K.J.: Optimal design of the head of a pneumatic pulsator. Chem. Eng. Trans. 18, 237-242 (2009). doi:10.3303/CET0918037

24. Utyuzhnikov, S.: Robin-type wall functions and their numerical implementation. Appl. Numer. Math. 58(10), 1521-1533 (2008)

25. Wernik, J., Wołosz, K.J.: Numerical simulations of air flow in a pneumatic pulsator. In: Conference Proceedings, pp. 9-12. IC-SCCE, Athens, Greece (2008)

26. Wernik, J., Wołosz, K.J.: Pneumatic pulsator design as an example of numerical simulations in engineering applications. Cent. Eur. J. Eng. 2(1), 76-82 (2012). doi:10.2478/s13531-011-0050-5

27. Wołosz, K.J., Wernik, J.: Three-dimensional flow optimization of a nozzle with a continuous adjoint. In: Buburuzan, A., Brindusa, R. Teodosiu, C. (eds.) Proceedings of the 7th International Conference Environmental Engineering and Management: Integration Challenges for Sustainability Conference Abstract Book, September, pp. 209-210. POLITEHNIUM Publishing House, Iasi (2013)

28. Yuan, W., Sauer, J., Schnerr, G.: Modeling and computation of unsteady cavitation flows in injection nozzles. Mécanique \& Industries 2(5), 383-394 (2001)

29. Zhu, Y., Cai, W., Wen, C., Li, Y.: Numerical investigation of geometry parameters for design of high performance ejectors. Appl. Therm. Eng. 29(5), 898-905 (2009). doi:10.1016/j.applthermaleng.2008.04.025

30. Zhu, Y., Jiang, P.: Experimental and analytical studies on the shock wave length in convergent and convergent-divergent nozzle ejectors. Energy Convers. Manag. 88, 907-914 (2014). doi:10.1016/j.enconman.2014.09.023 